\title{
Lutetium Lu 177-Edotreotide
}

National Cancer Institute

\section{Source}

National Cancer Institute. Lutetium Lu 177-Edotreotide. NCI Thesaurus. Code C88277.

A radioconjug ate consisting of the somatostatin analogue edotreotide labeled with lutetium Lu 177 with potential antineoplastic activities. Lutetium Lu 177-edotreotide binds to somatostatin receptors (SSTRs), with high affinity to type 2 SSTR, present on the cell membranes of many types of neuroendocrine tumor cells. Upon binding and internalization, this radioconjug ate specifically delivers a cytotoxic dose of beta radiation to SSTR-positive cells. Edotreotide is produced by substituting tyrosine for phenylalanine at the 3 position of the somatostatin analogue octreotide (Tyr3-octreotide or TOC) and chelated by the bifunctional, macrocyclic chelating agent dodecanetetraacetic acid (DOTA). 